\title{
Immuno-Dot Blot Analysis of a Monoclonal Antibody that Blocks the Human Monocyte Response to Migration Inhibitory Factor ${ }^{a}$
}

\author{
DAVID Y. LIU ${ }^{b, c}$ AND ROBERT F. TODD III ${ }^{d}$ \\ 'Department of Medicine \\ Harvard Medical School \\ Boston, Massachusetts \\ Department of Rheumatology and Immunology \\ Brigham and Women's Hospital \\ Boston, MA 02115 \\ ${ }^{d}$ Department of Internal Medicine \\ Simpson Memorial Institute \\ The University of Michigan Medical Center \\ Ann Arbor, Michigan 48109
}

Mononuclear phagocytes are critical for both the specific and nonspecific functions of the immune system. One aspect is the communication that occurs between mononuclear phagocytes and lymphocytes. This interaction proceeds by way of soluble mediators secreted by these cells, such as migration inhibitory factor (MIF). MIF is produced and secreted by activated lymphocytes and inhibits the migration of mononuclear phagocytes. We have previously established that membrane glycolipids of mononuclear phagocytes play a significant role in the regulation of the human monocyte response to MIF. ${ }^{1}$ Recently, a panel of murine monoclonal antibodies that recognize surface antigens specific for human cells of the mononuclear phagocyte lineage was developed by Todd and coworkers. ${ }^{2,3}$ These antibodies were used to further define the membrane components essential for the monocyte response to human MIF. We found that at least one monoclonal antibody (Mo3e) completely blocks the human monocyte response to MIF. ${ }^{4}$ This represents the first evidence for a monocyte-specific antigen that participates in a cellular function. The purpose of this study was to investigate by an immuno-dot analysis the nature of the antigen recognized by Mo3e.

Cells isolated from peripheral blood or carried as transformed cell lines were lysed in Tris-saline buffer containing NP-40 detergent, protease inhibitors, and iodoacetamide. The lysates were then centrifuged, and the supernatants serially diluted before being applied to nitrocellulose paper as spots in a 96-well suction apparatus (Schleicher and Schuell, Keene, NH). After blocking nonspecific binding sites on the paper, it was incubated sequentially with Mo3e, rabbit anti-

\footnotetext{
${ }^{a}$ This work was supported by National Institutes of Health Grants AI-07685, BRSG RR05669, CA39064-02, and National Science Foundation Grant PCM-8208262.

${ }^{b}$ Address for correspondence: Dr. David Y. Liu, Department of Immunology, Cetus Corporation, 3400 W. Bayshore Rd., Palo Alto, CA 94303.
} 
TABLE 1. Cellular Distribution of Human Mo3e Antigen

\begin{tabular}{lcccccc}
\hline Dilutions $^{a}$ & U937 & $\begin{array}{c}\text { U937 } \\
\text { + PMA }\end{array}$ & RBC $^{b}$ & $\mathrm{~T}^{c}$ & HL-60 & Mono $^{d}$ \\
\hline $1: 2$ & $18^{e}$ & 26 & 0 & 10 & 332 & 125 \\
$1: 4$ & 17 & 38 & 1 & 16 & 72 & 55 \\
$1: 8$ & 19 & 47 & 4 & 12 & 43 & 32 \\
$1: 16$ & 28 & 55 & 4 & 8 & 31 & 32 \\
$1: 32$ & 28 & 51 & 7 & 14 & 16 & 37 \\
$1: 64$ & 21 & 38 & 5 & 8 & 10 & 10 \\
$1: 128$ & 16 & 27 & 6 & 9 & 8 & 2 \\
\hline
\end{tabular}

${ }^{a}$ All cell lysate dilutions made from a $50 \times 10^{6}$ cell $/ 0.1 \mathrm{ml}$ original suspension.

${ }^{h} \mathrm{RBC}=$ erythrocyte.

$\mathbf{T}=$ cell hybridoma .

${ }^{d}$ Mono $=$ monocyte.

${ }^{e}$ Absorbance units after subtraction of background absorbance at $490-\mathrm{nm}$ wavelength of the ELISA reader.

TABLE 2. Effects of Heat, Chemical Reduction, and Proteases on Mo3e Antigen

\begin{tabular}{lcccccc}
\hline & \multicolumn{6}{c}{ Treatment of U937 Lysate } \\
\cline { 2 - 7 } Dilution & None & Heat $^{a}$ & Reduction $^{b}$ & $\begin{array}{c}\text { Heat and } \\
\text { Reduction }\end{array}$ & Trypsin $^{c}$ & Pronase $^{c}$ \\
\hline $1: 64$ & $131^{d}$ & 132 & 37 & 61 & 8 & 9 \\
$1: 128$ & 78 & 134 & 35 & 44 & 4 & 1 \\
$1: 256$ & 55 & 82 & 18 & 24 & 4 & 0 \\
$1: 512$ & 22 & 25 & 8 & 10 & 5 & 3 \\
\hline
\end{tabular}

${ }^{a} 100^{\circ} \mathrm{C} / 5 \mathrm{~min}$.

${ }^{b} 5 \%$ 2-mercaptoethanol for five minutes at room temperature.

${ }^{c} 100 \mu \mathrm{g} / \mathrm{ml}$ for 60 minutes at $37^{\circ} \mathrm{C}$.

${ }^{d}$ Absorbance units after subtraction of background absorbance at $490-\mathrm{nm}$ wavelength of the ELISA reader.

mouse IgM and ${ }^{125} \mathrm{I}$-labeled protein $\mathrm{A}$. This was followed by autoradiography and densitometric analysis on an ELISA reader.

As demonstrated in TABLE 1, Mo3e bound to lysates from human peripheral blood monocytes, histiocytic lymphoma cell line U-937 and the promyelocytic leukemic cell line HL-60. In contrast, Mo3e did not show binding to erythrocyte and T-lymphocyte hybridoma lysates. Stimulation of U-937 cells with $4 \beta$-phorbol 12-myristate 13-acetate (PMA) increased the expression of the Mo3e antigen, which is consistent with membrane cytofluorometric analysis of cells stimulated with PMA $^{3}$ and other activators of protein kinase $\mathrm{C}$, such as lipopolysaccharide and diacylglycerol (unpublished results).

The results of experiments represented by TABLE 2 show that the antigen from $\mathrm{U}-937$ cells recognized by the Mo3e antibody is resistant to heat $\left(100^{\circ} \mathrm{C} / 5 \mathrm{~min}\right)$ 
(column 1 versus column 2) and less resistant to treatment with 2-mercaptoethanol although still showing detectable binding (column 1 versus column 3; column 2 versus column 4). Furthermore, treatment of the lysate with trypsin or pronase resulted in no detectable binding, suggesting that the Mo3e, antigen is a protein.

The data presented here demonstrate that Mo3e, a monoclonal IgM antibody that blocks the human monocyte response to MIF, detects a protease-sensitive antigen that is associated with human peripheral blood monocytes and with myelocytic or histiocytic tumor cells. This suggests that the receptor for MIF may be a complex containing protein (Mo3e) and lipid ${ }^{5}$ components similar to the thyrotropin receptor complex. ${ }^{6}$

\section{ACKNOWLEDGMENTS}

We wish to express our appreciation to Joy Ree for assisting in this study and to Anne Armata for the preparation of this manuscript.

\section{REFERENCES}

1. LiU, D. Y., J. D. Forman \& C. G. Wilde. 1985. In Cellular and Molecular Biology of Lymphokines. C. Sorg \& A. Schimpl, Eds.: 335-339. Academic Press. New York.

2. TODD, R. F. III \& S. F. SCHLOSSMAN. 1982. Blood 59: 775-786.

3. TODD, R. F. III, P. A. Alvarez, D. A. BRoTt \& D. Y. LiU. 1985. J. Immunol. 135: 38693877.

4. LIU, D. Y. \& R. F. TODD III. 1986. J. Immunol. 137: 448-455.

5. LiU, D. Y., S. F. YU, P. A. Miller, H. G. Remold \& J. R. DAVID. 1984. Cell Immunol. 88: 350-360.

6. Valente, W. A. P. Vitti, Z. Yavin, E. Yavin, C. M. Rotella, E. F. Grollman, R. S. TOCCAFONDI \& L. D. KoHN. 1982. Proc. Natl. Acad. Sci. USA 79: 6680-6684. 